\title{
Introduction
}

\section{Michel DUMOULIN}

Les articles publiés dans cette livraison offrent l'occasion de souligner combien le passé et le présent sont intimement liés. Et combien, aussi, des questions qui, à un titre ou à un autre, font l'actualité du projet européen, peuvent être le reflet de préoccupations souvent anciennes.

En cette année du cinquantième anniversaire de la signature des traités de Rome, qui est également celle du débat sur la manière de sortir de la crise ouverte par le «non» des peuples français et néerlandais au projet de traité constitutionnel, Hungdah Su, d'une part, et Anne Boerger-De Smedt, d'autre part, offrent un article qui ne manque pas d'interpeller. Jean Monnet a narré son expérience chinoise entre 1933 et 1936 dans ses mémoires. Mais la recherche, qui a tellement produit de résultats au sujet de sa contribution à l'intégration européenne s'est fort peu arrêtée à ses activités internationales durant l'entre-deux-guerres. Parmi celles-ci, la création de la China Development Finance Corporation (C.D.F.C.) mérite de fixer l'attention, à la fois au point de vue de la biographie du personnage, mais aussi parce que ses contacts avec le gouvernement chinois en tant que cheville ouvrière d'une firme privée, Monnet, Murmane \& Co, renvoie à l'analyse de l'enjeu que représente, aujourd'hui, le rapport avec la Chine, tant au plan économique que politique. Une même actualisation de la problématique est présenté dans l'article que Boerger-De Smedt consacre aux origines de la composition de l'exécutif communautaire. En plus d'éclairer un point d'histoire institutionnelle, l'auteur donne du grain à moudre à tous ceux qui, aujourd'hui, dissertent longuement sur les adaptations que devrait subir la composition du collège des commissaires afin de rendre celui-ci plus efficace. Or, «derrière la valse des chiffres»- pour reprendre l'expression qui figure dans le titre même de l'article -, l'enjeu, hier comme aujourd'hui, est intensément politique puisqu'il met bien en lumière les sacrifices que les grands comme les petits Etats sont décidés à consentir ou non au plan de la représentation de leurs intérêts au sein de l'exécutif.

A cet égard, le cheminement du projet européen a mis en lumière combien, du noyau des Six constituant la «petite Europe» à l'Europe des 27 qui est actuellement la nôtre, chaque élargissement a été l'occasion d'un phénomène de décomposition/ recomposition de la vie communautaire causé par l'adhésion de nouveaux membres venant en quelque sorte bouleverser l'ordre ancien. A cet égard, l'article de Henning Türk relatif à l'attitude de la «grande coalition» au pouvoir à Bonn devant la deuxième demande d'adhésion britannique est significative. A force de se préoccuper principalement des positions françaises, l'historiographie a quelque peu négligé le point de vue des autres protagonistes de la négociation. Or, la nature même de la composition du gouvernement fédéral allemand rend l'analyse même de son attitude particulièrement intéressante dans la mesure où, a priori, elle est marquée par un compromis au sujet d'une demande britannique qui devait elle-même faire l'objet d'une approche consensuelle. 
Si la méthode communautaire, nourrie par l'esprit du consensus, a permis la progression du projet européen, il n'en demeure pas moins que, pour les uns, celui-ci ne va jamais assez loin tandis que, pour d'autres, il importe de freiner les ardeurs par trop intégrationnistes. A cet égard, les articles de David Burigana et de Christian Henrich-Franke fournissent deux excellents exemples appartenant tous deux au secteur de l'aéronautique civile. Au plan institutionnel tout d'abord, Henrich-Franke montre comment l'idée de créer une autorité aéronautique supranationale évolue vers celle de la mise en place de la Conférence européenne de l'aviation civile. A l'heure des fusions/acquisitions entre compagnies aériennes nationales dans le contexte de la libéralisation du transport aérien, cet article ne manque pas d'inviter à replacer le thème étudié dans le cadre général des finalités de la construction européenne. A Rome, en 1957, les Six laissent en effet la porte ouverte à la conception et à la mise en œuvre de politiques sectorielles que le traité ne prévoit pas. Or, malgré des visions de grand style, les exemples de blocage des ambitions de certains abondent. La politique industrielle reste improbable et la politique de la recherche est placée sous la sextuple tutelle des Etats. David Burigana en fournit la parfaite illustration en étudiant «le lancement d'Airbus et le sabordage d'une coopération aéronautique communautaire». Alors que la Commission européenne a lancé le 22 décembre 2006 les premiers appels pour la soumission de projets dans le cadre du $7^{\mathrm{e}}$ programme cadre (2007-2013) de recherche et de développement technologique, et que Airbus se débat dans les difficultés tant au point de vue du marché qu'à celui des rapports entre les partenaires français et allemand, la contribution sous rubrique dépasse de loin les seuls aspects techniques du dossier. En effet, les objectifs poursuivis en matière d'aéronautique civile soulèvent la question des rapports avec les Etats-Unis. Pour les premiers, l'enjeu est à la fois celui du marché et du contrôle de la technologie. Certes, ce dernier aspect est moins perceptible dans le domaine civil que dans le militaire où les Européens se voient contraints au milieu des années 1970 de revenir à des accords bilatéraux avec les Etats-Unis plutôt que de poursuivre dans la voie de projets communautaires. Mais certaines coïncidences ne sont pas fortuites. Confrontés au défi américain pour citer le titre d'un célèbre ouvrage de Jean-Jacques Servan-Schreiber, les Européens peinent à accorder leurs violons au moment où pointe l'ambition d'une stratégie des champions nationaux. Mais s'en tenir à ce seul facteur ne suffit pas. La relation trop souvent présentée d'une manière manichéenne entre l'Europe et les Etats-Unis doit impérativement être prise en compte. Les Etats-Unis sont tantôt un obstacle, tantôt un aiguillon du fait de leur attitude même. Ou bien les Européens plient devant la stratégie américaine et les liens de coopération se délient au profit d'un retour en force de relations bilatérales privilégiées entre Washington et chacune des capitales européennes ou bien la réaction s'organise. C'est le cas d'Airbus dont l'évolution à travers plusieurs décennies indique que les paramètres changeant, des adaptations sont indispensables.

Le caractère ambigu, voire même paradoxal en maintes occasions, des relations entre l'Europe et les Etats-Unis est illustré par l'article de Dimitri Grygowski 
consacré au bilan d'ensemble et aux perspectives de la recherche relative aux Etats-Unis et l'unification monétaire de l'Europe de 1968 jusqu'à 1998. Non sans insister sur les tensions internes qui existent au fil du temps au sein de l'administration américaine à propos des projets monétaires européens, l'auteur met en exergue «combien les perceptions américaines du projet de l'intégration européenne ont été conditionnées par les évolutions générales des politiques de sécurité dans le contexte de la guerre froide, mais aussi par les logiques autrement plus réelles des relations commerciales entre l'Europe et les Etats-Unis». En outre, et cela recoupe ce qui peut être observé sur le terrain de l'aéronautique, par exemple, l'Europe monétaire s'est trouvée confrontée à l'impact que les relations bilatérales entre Washington et tel pays européen - la République fédérale d'Allemagne notamment $-\mathrm{a}$ pu avoir. Mais il y a plus. Au fil du temps, d'où l'intérêt d'une lecture dans la durée, Washington paraît davantage privilégier la relation économique et financière avec d'autres régions du monde, à commencer par la Chine. Une hypothèse que sans rechercher à lier entre eux tous les articles de cette livraison, il importe de prendre en compte si l'on se tourne vers l'Afrique.

En effet, l'intérêt manifesté par Washington dans l'actuelle crise du Darfour ne s'explique pas uniquement par des préoccupations de nature humanitaire mais bien par la volonté de contrer une influence chinoise de plus en plus manifeste au Soudan notamment. Or, l'enjeu que représente l'Afrique est loin d'être neuf. L'association, inscrite dans le traité CEE, a conduit l'Europe des Six à mettre en place une politique de l'aide au développement qui révéla le rôle que la France entendait y jouer avant que l'adhésion britannique ne confirme un changement d'orientation au bénéfice d'autres régions que l'Afrique noire francophone. Mais dès 1958, comme l'expose Guia Migani, le projet européen qui ne va pas sans créer des tensions entre la France et ses partenaires, entre aussi en compétition, dans le contexte du rapide mouvement de décolonisation qui se déroule, avec la manière de voir de la Commission économique pour l'Afrique de l'ONU. A un projet européen conçu et porté par des puissances qui ne sont plus coloniales que pour fort peu de temps répond une prétention plus universelle et davantage marquée par les préoccupations d'un Tiers Monde objet de l'attention des Etats-Unis et de l'URSS.

Les articles publiés ici invitent bel et bien au travail de va et vient entre passé et présent dont Marc Bloch disait l'impérieuse nécessité. Etant aussi, majoritairement, le fruit du travail de recherche de jeunes historiens, ils constituent un gage précieux de l'enrichissement d'une historiographie qui se diversifie toujours davantage à propos du phénomène complexe et jamais achevé qu'est la construction européenne. 


\section{Deutsche Interessenvertretung in Europa}

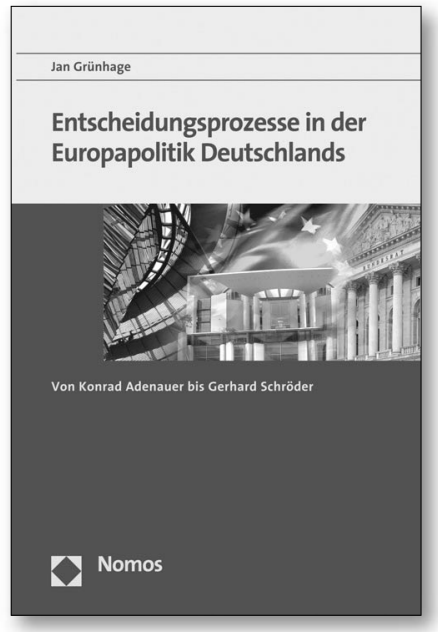

\section{Entscheidungsprozesse in der Europapolitik} Deutschlands

Von Konrad Adenauer bis Gerhard Schröder Von Jan Grünhage 2007, 389 S., brosch., 49,- $€$, ISBN 978-3-8329-2705-9
Die Untersuchung zeigt, dass die Ausgestaltung der europapolitischen Entscheidungsprozesse in Deutschland die deutsche Interessenvertretung in Europa erschwert. Die gewonnenen Erkenntnisse beruhen u.a. auf Interviews mit Praktikern und werden durch eine Fallstudie ergänzt.

In dem Werk werden zahlreiche praktische Wege zur Erhöhung der Schlagkraft der deutschen Europapolitik aufgezeigt. Es ist für Wissenschaftler, Vertreter der Wirtschaft und der Verbände ebenso von Interesse wie für die europapolitischen Entscheidungsträger in der Bundesregierung, im Bundestag und in den Ländern.

Der Autor hat u.a. am Europakolleg Brügge studiert und ist in der Europaabteilung des Bundeskanzleramtes beschäftigt. 